\section{птим льное упорядочение конфликтующих объектов и 3 д ч коммивояжер}

\author{
оеводин, . осяченко \\ Silver-AVV@yandex.ru, spiero@yandex.ru \\ идео нтернешнл
}

ннот ция. $\mathrm{p}$ боте предст влен пост новк 3 д чи оптим льного упорядочения конфликтуюших объектов и ее связь с $з$ д чей коммивояжер (Travelling Salesman Problem или TSP). д ч оптим льного упорядочения конфликтующих объектов возник ет в социологии, при н лизе гр фов в соци льных сетях, при р змещении рекл мных 3 к зов в сетях - ст тье опис ны используемые втор ми н пр ктике быстрые лгоритмы решения этой и связ нных с ней 3 д ч. кже p ссмотрен 3 д ч TSP c p зреженной м трицей штр фов. ля з д ч TSP c ленточной и блочно-ди гон льной м триц ми н йдены необходимые и дост точные условия и построены точные лгоритмы, при которых достиг ется нулевое миним льное зн чение целевой функции з д чи. редложены эффективные лгоритмы и для произвольных $\mathrm{p}$ зреженных м триц. риведены результ ты н литических и численных исследов ний сложности $\mathfrak{p}$ зр бот нных лгоритмов и точности решения,

т кже рекоменд ции по применению лгоритмов для решения з д ч подобного тип

лючевые слов : оптим льное $\mathrm{p}$ змещение; $з$ д ч коммивояжер ; TSP; NP-трудные 3 д чи; ленточные м трицы; р зреженные м трицы; ж дный лгоритм; штр фн я функция; конфликты, сети

\section{ведение}

д ч оптим льного р змещения конфликтующих объектов обобщ ет $з$ д чу коммивояжер , т кие з д чи возник ют во многих предметных обл стях. то кл ссическ я з д ч коммивояжер обход всех городов по кр тч йшему пути [1], 3 д ч о сверлильном ст нке, 3 д ч о производстве кр сок, пополнение 6 нком тов н личными деньг ми, сбор сотрудников в хтовым методом, р склейк фиш, другие з д чи логистики [2]. общем случ е в p ссм трив емой н ми з д че не все объекты связ ны и конфликтуют между собой, н зн ч емые 3 конфликт штр фы могут быть большими при непосредстенном соседстве объектов и уменьш ются при отд лении их друг от друг в списке. ля з д ч коммивояжер обычно сушествует один тип штр ф, н пример: р сстояние, стоимость или время дост вки груз между город ми. общем случ е может быть несколько типов штр фов, определяемых р зными причин ми нежел тельного соседств объектов, н пример, прин длежностью их к конфликтующим групп м по р зличным призн к м. кие з д чи возник ют в социологии, при н хождении оптим льных путей между вершин ми гр ф в соци льных сетях, при р змещении рекл мных $3 \mathrm{~K}$ зов в сетях . есмотря н то, что д нн я 3 д ч в общем случ е относится к кл ссу NP-трудных з д ч, для н иболее p спростр ненных ч стных случ ев 3 д чи TSP c p зреженной м трицей штр фов построены точные быстрые лгоритмы. ля общего случ $я$ предложен эвристический быстрый лгоритм, м сшт бируемый до произвольного р змер при сохр нении линейной сложности при незн чительных потерях в к честве решения. то позволяет н м пр ктически применять предложенные лгоритмы н больших объем х д нных.

\section{ост новк 3 д чи и ее связь с 3 д чей коммивояжер}

но неупорядоченное множество из $N$ объектов $x_{i}, i=1, \ldots, N$. ребуется упорядочить эти объекты в виде линейного списка. Объекты «конфликтуют» между собой, конфликтующие объекты жел тельно разместить в списке под льше друг от друга. Математически конфликт выражается в том, что зі непосредственное соседство в списке объектов $x_{i}$ и $x_{j}$ применяется штраф $p_{i j} \geq 0$. сли же между $x_{i}$ и $x_{j}$ в списке н ходится сще один объект $x_{k}$, то штр ф з соседство $x_{i}$ и $x_{j}$ уменьшается в $2 \mathrm{p} 3$. сли между $x_{i}$ и $x_{j}$ в списке находятся два других объекта, то штраф з соседство $x_{i}$ и $x_{j}$ уменьшается в 3 раза и т.д. В общем случае если после упорядочения в списке между объект ми $x_{i}$ и $x_{j}$ оказалось $L_{i j} \geq 0$ других объектов, то з т кую близость $x_{i}$ $x_{j}$ применяется штраф, равный $\frac{p_{i j}}{L_{i j}+1}$. К примеру, при $N=4$ перест новке объектов $x_{3}, x_{1}, x_{4}, x_{2}$ будет соответствовать суммарный штраф

$$
W=p_{31}+p_{14}+p_{42}+\left(p_{34}+p_{12}\right) / 2+p_{32} / 3 \text {. }
$$

ребуется среди всех перест нновок объектов н йти перестановку с миним льной величиной штр $ф W$. ๔орм льно пост новка задачи выглядит следующим обр зом. меется множество из $N$ объектов $x_{1}, x_{2}, \ldots, x_{N}$. На исходной нумер ции этих объектов зад ется м триц штр фов $P$ с элементами $p_{i j} \geq 0$ за непосредственное соседство в списке объектов $x_{i}$ и $x_{j}$. Пусть $\mathrm{S}-$ множество всех перестановок $s_{k}$ чисел $1,2, \ldots, N$, где $k=1, \ldots, N !$. Обозн чим $L_{i j}^{k} \geq 0$ - количество объектов из исходного множеств, ок з вшихся в перестановке $s_{k}$ между объект ми $x_{i}$ и $x_{j}$. Необходимо среди всех перест новок $s_{k}$ объектов н йти перестановку с миним льным сумм рным штр фом $W_{k}$ : 


$$
\min _{k} W_{k}=\min _{k} \sum_{i=1}^{N-1} \sum_{j=i+1}^{N} \frac{p_{i j}}{L_{i j}^{k}+1}
$$

ч стном случ е, когда штрафуется только непосредственное соседство объектов, все зн чения $L_{i j}^{k}=0$, и штр ф $p_{i j}$ применяется только $N-1 \mathrm{p} 3$ к непосредственно соседствующим объектам. этом случ е 3 д чу (1) упорядочения конфликтующих объектов можно з пис ть в виде

$$
\min _{k} w_{k}^{*}=\min _{k} \sum_{i=1}^{N=i+1} p_{i, i+1}
$$

где минимум берется по всем $N$ ! перест новк м $s_{k}$ индексов объектов $x_{i}, i=$ $1,2, \ldots, N$.

птимиз ционн я $з$ д ч (2) является кл ссической з д чей TSP, котор я в теории сложности относится к кл ссу NP-трудных 3 д ч. ля метрических 3 д ч TSP существуют приближенные лгоритмы полиноми льной сложности, которые г р нтиров нно н ходят решение м ксимум вдвое отлич ющееся от оптим льного[1]. ссм трив ем я н ми 3 д ч (2) относится к в ри нту нез мкнутой, симметричной, неметрической $з$ д чи TSP, для которых пок не предложено приближенных лгоритмов полиноми льной сложности с хорошей оценкой точности. ким обр зом, в общем случ е для з д ч (1) и (2) не приходится р ссчитыв ть н существов ние полиноми льно быстрых и точных лгоритмов решения. дн ко для ряд $\mathrm{p}$ спростр ненных н пр ктике случ ев н м уд лось это сдел ть.

метим, что хотя 3 д ч (2) оптим льного упорядочения конфликтующих объектов форм льно относится к 3 д че TSP, ф ктически он отлич ется от кл ссической $з$ д чи коммивояжер н хождения оптим льного пути обход городов н плоскости и требует построения специальных алгоритмов. же метрическую $з$ д чу TSP не всегда можно изобразить на плоскости к к 3 д чу обход городов. пример, р ссмотрим задачу с $N$ рєвноудаленными друг от друг вершин ми, т.е. все $p_{i j}=C>0, i \neq j$, где $C$-константа. кое множество вершин можно изобразить на плоскости только при $N=3$. общем случ е $N$ р вноуд ленных вершин потребуют не менее $(N-1)$-мерного простр нств, где обр зуют $(N-1)$-мерный регулярный симплекс [3]. еметрическ я $з$ д ч TSP, к которой относится р ссм трив ем я н ми з д ч (2), еще менее похож н кл ссическую 3 д чу коммивояжер. ней не выполняется нер венство треугольник, ее можно предст вить только в виде взвешенного гр ф или в м тричном виде.

о с мое большое отличие 3 д чи оптим льного упорядочения конфликтующих объектов (2) от кл ссической 3 д чи коммивояжер 3 ключ ется в том, что не к ждый объект конфликтует с к ждым, т.е. в м трице штр фов некоторые зн чения $p_{i j}=0$. Еолее того, взвешенный гр ф 3 д чи (2) может быть несвяз нным и может расп д ться н несвяз нные друг с другом компоненты. оскольку н ш задача неметрическ я, то стоимость «обходного» пути между вершин ми $i$ и $j$ может быть м лой или д же нулевой: $p_{i, k}+p_{k j}=0, \quad$ стоимость «непосредственного» пути по дуге $p_{i j}$ большой. Когд в в м трице штр фов много нулевых элементов $p_{i j}$, он н зыв ется р зреженной. о несмотря н все эти отличия от кл ссической з д чи коммивояжер з д ч упорядочения конфликтующих объектов (2) ост ется 3 д чей TSP и д же может иметь смысловое н полнение близкое к кл ссическому. триц штр фов может озн ч ть стоимость дополнительных, не входящих в б зовый н бор, услуг н пути обход городов, н пример: стоимость проезд по пл тным дорог м, опл т номеров и п рковки в отелях повышенного кл сс н тр ссе и т.п. к я м триц может быть $\mathrm{p}$ зреженной. д нной ст тье мы построим лгоритмы, эффективные для 3 д ч TSP c p зреженной м трицей штр фов. удут предложены процедуры сведения $з$ д чи с х отически р зреженной м трицей к 3 д ч м с ленточной или блочно-ди гон льной м трицей. свою очередь для 3 д ч TSP c ленточной или блочно-ди гон льной м триц ми будут н йдены необходимые и дост точные условия и построены лгоритмы, при которых всегд существует путь с $W^{*}=0$. жные и ч сто встреч ющиеся н пр ктике кл ссы з д ч с ленточной и блочно-ди гон льной м триц ми р ссмотрены в р боте отдельно.

\section{тричн Я пОст НОвК 3 д чи}

м тричном виде $з$ д ч (2) сводится к одноименной перестановке строк и столбцов симметричной неотриц тельной м трицы стоимости $P=\left(p_{i j}\right), p_{i j} \geq$ 0 , т к, чтобы сумм чисел н 2-й ди гон ли, н ходяшейся непосредственно н д гл вной, был миним льн . юб я одноименн я перест новк строк и столбцов м трицы эквив лентн преобр зов нию $T * P * T$, где $T-$ м триц перест новок, $T^{t}$ - тр нспониров нн я к ней м триц . триц перест новок это 0-1 м триц, у которой в любой строке и любом столбце только один ненулевой элемент. еремножение м триц перест новок снов д ет м трицу перест новок. триц $T^{t}$ т кже является м трицей перест новок. множение слев м трицы $P$ н м трицу $T$ приводит к перест новке строк м трицы $P$. множение спр в м трицы $P$ н м трицу $T^{t}$ приводит к одноименной перест новке столбцов м трицы $P . \quad$ триц $T^{*} P^{*} T^{t}$ снов будет симметричной м трицей. результ те одноименных перест новок строк и столбцов м трицы $P$ ее ди гон льные элементы будут ост в ться н гл вной ди гон ли. Ким обр зом, м тричн я пост новк 3 д чи (2) следующ я.

йти м трицу перест новок $T$ т кую, чтобы у м трицы $T^{*} P^{*} T^{t}$ сумм чисел н 2-й ди гон ли был миним льн . 


\section{птим льный лгоритм для 3 д чи с блочно-} ди гон льной м трицей

пределение. триц, котор я перест новкой одноименных строк и столбцов может быть приведен к блочно-треугольному виду, н зыв ется р зложимой.

н шем случ е симметричной м трицы р зложим я м триц приводится очевидно к блочно-ди гон льному виду. имметричн я м триц $P$ р зложим тогд и только тогд , когд ее гр фнесвязен. Јправедлив следующ я

емм 1. сли м триц $P$ порядк $N$ р зложима и максим льный р змер блок в ее блочно-ди гольном предст влении $\leq\left[\frac{N}{2}\right]$, то минимум в 3 д че (2) оптим льного упорядочения объектов р вен 0 .

риведем лгоритм, док зыв ющий лемму 1 и обеспечив ющий оптим льное решение $з$ д чи (2).

лгоритм 1. птим льное упорядочение строится следующим обр зом. н ч л в списке поочередно р спол г ем все первые вершины из к ждого блок м трищы, 3 тем вторые вершины из к ждого блок! м трицы и т.д. оскольку при т ком лгоритме любые две рядом стоящие в списке вершины $i$ и $j$ будут прин длеж ть р зным ди гон льным блок м м трицы, они не будут связ ны между собой, т.е. для любых соседних вершин $p_{i j}=0, \quad$ зн чит зн чение $W^{*}$ суммы в $з$ д че (2) равно 0 . лгоритм требует $N-1$ ш гов.

ледствие. Ғсли гр фз д чи (2) несвязен, и м ксим льное число вершин в связной компоненте гр ф.. $\leq\left[\frac{N}{2}\right]$, то минимум в $з$ д че (2) оптим льного упорядочения объектов р вен 0 .

тметим, что если в условиях леммы 1 использов ть поп рные перест новки вершин, то потребуется $\leq\left[\frac{N}{2}\right]$ поп рных перест новок. ерест новке вершин $i$ и $j$ соответствуют поп рные одноименные перест новки $i$-й и $j$-й строк и $i$-го и $j$-го столбцов м трицы $P$. любой поп рной одноименной перест новке строк и столбцов среди интересуюших н с элементов 2-й ди гон ли м тришы $P$ меняются только 4 элемента н $:$ новые. Гочнее при перестановке вершин $i$ и $j$ элементы меняются следующим образом:

$p_{i-1, i} \leftrightarrow p_{i-1, j}, p_{i, i+1} \leftrightarrow p_{i+1, j}, p_{j-1, j} \leftrightarrow p_{i, j-1}, p_{j, j+1} \leftrightarrow p_{i, j+1}$.

десь мы учитыв ем,пnето матриц $P$ симметричн я. ким обр зом, при поп рной перест новкшдостаточно в формуле суммы целевого функцион л (2) подменить только : выходит из ди п зон $\overline{i j i j} \bar{v}$, то соответствующий элемент не перест вляется и в сумме не уч ствует.

лгоритм 1, обеспечив ющий нулевой минимум в 3 д че (2) для р зложимой м трицы и ее несвяз нного гр ф, можно успешно применять в к честве приближенного лгоритм к м триц м близким к блочно-ди гон льным, которых все элементы, н ходящиеся вне ди гон льных блоков, м лы относительно элементов внутри ди гон льных блоков.

\section{птим льные}

\section{м трицей}

ругим в жным кл ссом м триц, для которых можно построить лгоритм с нулевым зн чением штр ф в задєче (2), является кл сс ленточных симметричных м триц.

пределение. $\wedge$ трица $P$ порядк $N \geq 2$ н зывается ленточной, если

$$
p_{i j}=0, \quad \text { при }|i-j| \geq l,
$$

еличин $l>0$ н зыв ется полушириной ленты т кой м трицы.

пример, трехди гон льн я м триц будет иметь полуширину ленты $l=2$. помним, что в р ссм трив емых н ми 3 д ч х (1) и (2) м трица $P$ симметричная.

емм 2. Если матриц $P$ порядк $N$ является ленточной с полушириной ленты $l \leq\left[\frac{N}{2}\right]-1$, то минимум в 3 д че (2) оптим льного упорядочения объектов равен 0 .

риведем лгоритм поп рных перест новок строк и столбцов, обеспечив ющий утверждение леммы 2.

лгоритм $2 . \quad$ ля $N \leq 5$ утверждение очевидно, поскольку тогд $l \leq 1$ и м триц $P$ имеет нулевую 2-ю ди гональ. Пусть $N>5$. ндексы $i, j$ попарных одноименных перест новок строк и столбцов будем бр ть к к индексы элементов м трицы $P$, леж щие н เ ее $\left(\left[\frac{N}{2}\right]+1\right)$ - й ди гон ли. р ть следует индексы не всех элементов с этой диагонали, а герез один, н чин я с 1-го. исло т ких элементов будет $\left\lceil\frac{N}{4}\right\rceil$. Поп рные перест новки выполняем последов тельно в соОгветствии с индекс: ми ук з нных элементов, н чин я с 1-го верхнего элемента д нной ди гон ли. ри перест новк х вершин $i$ и используем формулы (3). з них видно, что мы последов тельно перест вляем четверки соседних с $p_{i j}$ нулевых элементов, н ходящихся вне ленты м трицы, н ее 2-ю ди гон ль.

тметим, что при поп рных одноименных перестановк х строк и столбцов с индекс ми $i, j$ ленточной м трицы происходит з полнение элементов матриць в $i$-й строке и $j$-м столбце, отстоящих от $p_{i j} \mathrm{H} \leq l-1$ позиций. Но этот факт не меш ет р боте д нного лгоритм, поскольку индексы с $\left(\left[\frac{N}{2}\right]+1\right)-$ й ди гон ли м трицы $P$ берутся через один. лгоритм 2 требует $\left[\frac{N}{4}\right]$ поп рных перест новок одноименных строк и столбцов м трицы. 
гр ничение н полуширину ленты в лемме 2 можно осл бить, если использовать алгоритм с бо́льшим числом перест новок. именно, спр ведливо следующее утверждение.

емм। 3. Если м триц $P$ порядк $N$ является ленточной с полушириной ленты $l \leq\left[\frac{N}{2}\right]$, то минимум в з д че (2) оптим льного упорядочения объектов р вен 0 .

риведем лгоритм, обеспечив ющий утверждение леммы 3.

лгоритм 3. птим льное упорядочение строится следующим обр зом. ссмотрим упорядочение вершин $1,2, \ldots N, 3$ д в емое исходной ленточной м трицей. оптим льном списке р спол г ем эти вершины подряд в к ждую четную позицию, н чин я со 2-й позиции. Достигнув конца списк , вершинь р спол г ем подряд в к ждую нечетную позицию, н чиная с 1-й позиции. птим льное упорядочение построено. Іапример, для $N=8$ м ксим льн полуширин ленты м трицы $P$ будет $l=4$. птим льное упорядочение вершин будет т ким: $5,1,6,2,7,3,8,4$. кое упорядочение обеспечив ет нулевой минимум в 3 д че (2), ибо из структуры исходной ленточной м триць следует, что в построенном оптим льном списке любые две рядом стоящис вершины $i$ и $j$ не связдғы между собой, т к к к для них $p_{i j}=0$. ак, в рассматриваемом примере в исходной ленточной м трице элементы $p_{51}=$ $p_{16}=p_{62}=p_{27}=p_{73}=p_{38}=p_{84}=0$. н чит, для ук 3 нного оптим льного списка вершин 5,1,6,2,7,3,8,4 минимум в 3 д че (2) р вен 0 . лгоритм 3 требует $N-1$ ш гов - примерно в 4 р 3 больше, чем лгоритм 2 .

ледствие. сли для графа зад чи (2) существует упорядочение вершин, при котором люб я вершин। в списке связ н только с вершин ми, отстоящими от нее не более, чем н $\left[\frac{N}{2}\right]-1$ позиций в списке, то минимум в 3 д че (2) оптим льного упорядочения объектов р вен 0 .

ействительно, т кое упорядочение вершин описыв ется ленточной м трицей с полушириной ленты $\leq\left[\frac{N}{2}\right]$. Значит, в силу леммы $3, W^{*}=0$.

тметим, что ук з нное в лемме 3 огр ничение н полуширину ленты $\leq\left[\frac{N}{2}\right]$ является предельным. сли полуширин ленты превыш ет это зн чение, то нулевсе зн чение функцион л 3 д чи (2) г р нтиров ть уже нельзя. очнее спр вед.тив следующ я

еорем: 1. усть $P$ - симметричная лснточн я м триц порядк $N$, у которой все $p_{i j}>0(i \neq j)$ в предел х ее ленты. Іля того чтобы минимум в 3 д че $(2)$ оптимального упорядочения объектов был р вен 0 , необходимо и дост точно, чтобы полуширин ее ленты $l \leq\left[\frac{N}{2}\right]$. ок $з$ тельство. Дост точность условия следует из леммы 3 . ок жем необходимость от противного: если $l>\left[\frac{N}{2}\right]$, то $W^{*}>0$. усть полуширин ленты $l \geq\left[\frac{N}{2}\right]+1$. Тогд , поскольку ширина ленты $L=2 * l-1$, получ ем, что $L \geq 2 *\left[\frac{N}{2}\right]+1 \geq N$. То есть ширин ленты $L=N$. А по условиям теоремы все $p_{i j}>0(i \neq j)$ в предел х ленты. н чит в мтрице $P$ существует строк с некоторым номером $k$, в которой все ее элементы $p_{k j}>0$ для $j \neq k$ от 1 до $N$, и столбец с некоторым номером $k$, в котором все его элементы $p_{i k}>0$ для $i \not k$ от 1 до $N$. ругими слов ми вершин с номером $k$ конфликтует со всеми другими вершин ми. поскольку при любых перест новк х вершин с номером $k$ будет соседствовать хотя бы с одной другой вершиной, то для фунцион л в з д че (2) будет спрі ведлива оценк

$$
W^{*} \geq \min _{i \neq k} p_{i k}>0,
$$

что и требов лось док з ть.

лгоритмы 2 и 3, обеспечив ющие нулевой минимум в 3 д че (2), можно успешно применять в к честве приближенных лгоритмов к м триц м близким к ленточным, у которой все элементы, н ходящиеся вне ее ленты, м лы относительно элементов внутри ленты. ля приведения м трицы к ленточной или близкой $\mathrm{K}$ ленточной можно применять специ льные лгоритмы.

лгоритмы для 3 д ч с р зреженными и м триц ми общего вид

ля случ я р зреженных м триц общего вид, когд в м трице $P$ ненулевые элементы $\mathrm{p}$ сположены $\mathrm{x}$ отично, можно использов ть эффективные методы группиров ния ненулевых элементов н ди гон лях близких к гл вной.

пример, это прямой и обр тный методы тхилл- кки для р зреженных м триц, которые с помошью одноименных перест новок строк и столбцов приводят м трицу к ленточному виду с шириной ленты зн чительно меньшей, чем у исходной. ложность этой процедуры $O\left(N^{2}\right)[4] . \quad$ р бот х $[4,5]$ приводятся многочисленные примеры, когд , применяя прямой или обр тный методы тхилл- кки к р зреженной м трице р змер $N$, получ ем ленточную м трицу с шириной ленты в несколько р з или д же н порядок меньше, чем у исходной м трицы. согл сно теореме 1 для достижения нулевого минимума функцион л дост точно всего лишь, чтобы полуширин ленты был $\leq\left[\frac{N}{2}\right]$. В этом случ е, после процедуры тхилл- кки следует применить лгоритмы 2 или 3 оптим льного упорядочения для достижения нулевого минимум функцион л 3 д чи (2). 
тот же подход можно успешно применять и в случ ях, когд м триц $P$ не является р зреженной, то есть $P-$ м триц общего вид, но в ней явно выделяются большие и м лые элементы. ля м триц т кого вид т кже сн ч л используем методы тхилл- кки для группиров ния больших элементов н ди гон лях близких к гл вной. тем применяем лгоритмы 2 или 3 оптим льного упорядочения для минимиз ции функцион л 3 д чи (2).

ким обр зом мы построили новый быстрый алгоритм приближенного решения кл ссической общей з д чи TSP. тот Ілгоритм будет дост точно точно р бот ть в тех случ ях, когд элементы $p_{i j}$ в м трице штр фов $P$ дост точно сильно отлич ются по величине друг от друг, число «м лых» элементов в м трице не меньше $N^{2} / 4$.

\section{лгоритмы для 3 д чи с м трицей общего вид}

есмотря н существов ние оптим льных быстрых лгоритмов для блочноди гон льных, ленточных и р зреженных м триц, т кже эффективных лгоритмов для близким к ним м триц, в общем случ е в з д ч х (1) и (2) не приходится р ссчитыв ть н дост точно точные лгоритмы полиноми льной сложности. ложность лгоритм полного перебор сост вит $O(N !)$. сли для м лых $N$ выполнение полного перебор возможно, то с ростом $N$ мы столкнёмся с проблемой нехв тки вычислительных ресурсов. гр ничения в вычислительных ресурс $\mathrm{x}$ приводят $\mathrm{K}$ необходимости построения экономичного в вычислительном смысле лгоритм поиск решения, близкого к оптим льному.

рименим к решению 3 д ч “ж дный” лгоритм [6] (greedy algorithm), в котором к ждый ш г является лок льно оптим льным. . иссмотрим р боту ж дного лгоритм для пост вленной выше 3 д чи (1) размещения $\mathrm{H}$ примере.

лгоритм 4. усть есть множество объектов $\left(x_{1}, x_{2}, x_{3}, x_{4}\right)$. триц поп рных штр фов имеет вид: $P=\left|\begin{array}{llll}0 & 1 & 2 & 2 \\ 1 & 0 & 3 & 3 \\ 2 & 3 & 0 & 1 \\ 2 & 3 & 1 & 0\end{array}\right|$.

к к к в р боте ж дного лгоритм н м необходимо н чин ть р змещение с с мых конфликтных объектов, упорядочим объекты в порядке убыв ния «конфликтности», которую определим кик сумму элементов соответствующей строки м трицы $P$. олучим вектор конфликтности $\bar{p}=(5,7,6,6)$. соответствии с ним будем р змещ ть объекты в порядке $\left(x_{2}, x_{3}, x_{4}, x_{1}\right)$.

щем оптим льное $\mathrm{p}$ змещение для $x_{2}$, ст вим $x_{2}$ в первую доступную позицию:

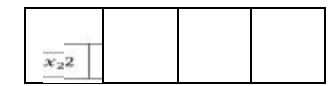

учетом уже пост вленного $x_{2}$ ищем оптим льное $\mathrm{p}$ змещение для $x_{3}$ :

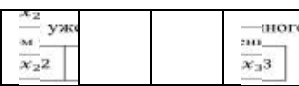

учетом уже пост вленных $x_{2}$ и $x_{3}$ ищем оптим льное $\mathrm{p}$ змещение для $x_{4}$ :

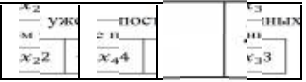

конец, ст вим $x_{1}$ в последнюю доступную позицию:

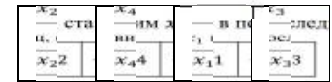

олуч ем штр $\phi W=9$.

сновным недост тком «ж дного» лгоритм является оптимиз ция только одного следующего ш г. ля его улучшения вторы предл г ют воспользов ться следующим эвристическим лгоритмом.

лгоритм 5.

1. с сли $N \unlhd$, то выполняем оптимальное размещение полным перебором и н выход. н че переходим к шагу 2.

2. се объекты сортируютсs в порядке убыв ния «конфликтности». ронумеруем их заново $\left(x_{1}, x_{2}, \ldots, x_{N}\right)$. Смещение $S=0$. писок $\mathrm{p}$ змещения - пустой.

3. ерем 7 объектов $\left(x_{S+1}, \ldots, x_{S+7}\right)$ и с помощью полного перебор ищем оптим льное р змещение этого подмножеств в списке.

4. Если $S+7=N$, то все объекты р змешены - ыход.

5. Из всех р змещенных 7 объектов ост вляем в списке только первый $x_{s+1}$, ост льные убир ем.

6. $S=S+1$. озвр щ емся к ш гу 2.

тличием лгоритм 5 от приведенного выше жадного алгоритм 4 3 ключ ется в том, что оптим льное р змещение $x_{S+1}$ ищется не лок льно оптим льно, с учетом других конфликтующих объектов, что позволяет лучше определять оптим льную позицию для $x_{S+1}$

сли лгоритм 5 применить к вышеприведенному примеру, то выполняя полный перебор д же для 2 объектов вместо 7, получим оптим льное зн чение сумм рного штр ф $W=7.5$.

д нном случ е н м уд лось добиться глоб льного минимум , хотя понятно, что в общем случ е д нный лгоритм н т кой результ т не претендует.

к пок зыв ет пр ктик, предложенный лгоритм 5 удовлетворительно реш ет 3 д чи р змером $N \leq 30$. ри $N>30$ скорость р боты лгоритм н чин ет 3 метно п д ть. ля компенс ции этого недост тк для больших $N$ 
предл г ется р збив ть позиции списк н подблоки т ким обр зом, чтобы длин к ждого подблок был не более 30 , и проводить оптимиз цию в к ждом подблоке «почти» отдельно.

лгоритм 6. Имеем $N>30$ объектов, они отсортиров ны в порядке убыв: ния штр ф $\left(x_{1}, x_{2}, \ldots, x_{N}\right)$. Вычисляем количество подблоков $K=\left[\frac{N}{30}\right\rceil$. Начин เя с первого объекта, распределяем последов тельно все объекты $\left(x_{1}, \ldots, x_{N}\right)$ поочередно по подблок м $1, \ldots, K$. огд подблоки конч тся, р спределяем объекты с последнего подблок до первого, з тем снов с первого до последнего и т.д. $\quad$ ким обр зом, в к ждом подблоке мы получим примерно один ковый по конфликтности н бор объектов. осле т кого р збиения предложенный выше лгоритм р змещения применяем к к ждому подблоку отдельно. для того, чтобы избеж ть р змещения конфликтующих объектов н гр нице подблоков будем применять приведенный выше лгоритм еще и н гр нице подблоков следующим обр зом.

ссмотрим пример. усть имеется 2 подблок. се множество объектов соответственно поделено н 2 группы объектов, которые должны быть р змещены в подблок х 1 и 2 соответственно. н ч л выполняется размещение в 1-м подблоке. тем р змеш ем объекты 2-ого подблок учетом р змещения 1-ого подблок . тем выделяем промежуточный подблок на границе 1-ого и 2-ого подблок . д нный промежугочный подблок входят $\left[\frac{l 1}{2}\right]$ последних объектов 1-ого подблок и $\left[\frac{l 2}{2}\right]$ первых объектов 2-ого подблок, где $l 1, l 2$ - количество объектов в 1-ом и во 2-ом подблоке соответственно. ыполняем размещение в промежуточном подблоке с учетом р сст новки первых $l 1-\left[\frac{l 1}{2}\right]$ объектов 1-ого подблок. лее еще р 3 проводим перер змещение объектов во 2-ом подблоке. ким обр зом, уд ется улучшить $\mathrm{p}$ змещение н гр нице подблоков. метим, что результ р боты предложенного лгоритм 6 может быть д лек от оптим льного, поэтому для конечной «шлифовки» будем дополнительно использов ть лгоритм лок льной оптимиз ции, приведенный ниже.

\section{лгоритм лок льной оптимизации с помощью поп рных перест новок}

удем менять мест ми все п ры вершин $\left(x_{i}, x_{j}\right)$, если это приводит к уменьшению . к поступ ем до тех пор, пока происходит уменьшение ожно т кже н к ждом ш ге выбир ть н илучшую перест новку вершин $i$ и $j$, у которой (сумм ст рых 4 элементов 2-й ди гон ли) - (сумм новых 4 элементов 2-й ди гон ли) м ксим льн . ри этом учитыв ем, что в любой поп рной одноименной перест новке строк и столбцов среди интересуюших н с элементов 2-й ди гон ли м трицы $P$ меняются только 4 ст рых элемент н новые. ндексы ст рых и новых элементов определяются формул ми (3). к поступ ем, пок н илучш я перест новк будет д в ть выигрыш по функцион лу. кой метод сходится к лок льному минимуму з конечное число ш гов. ложность одного ш г $O\left(N^{2}\right)$. сли т кую процедуру поп рной перест новки применять только внутри к ждого подблок , то общ сложность всех поп рных перест новок и предложенного лгоритм упорядочения всех конфликтующих объектов в целом будет р вн $O(N)$.

бщ я схем функциониров ния предложенного лгоритм приведен $\mathrm{H}$ рисунке 1 .

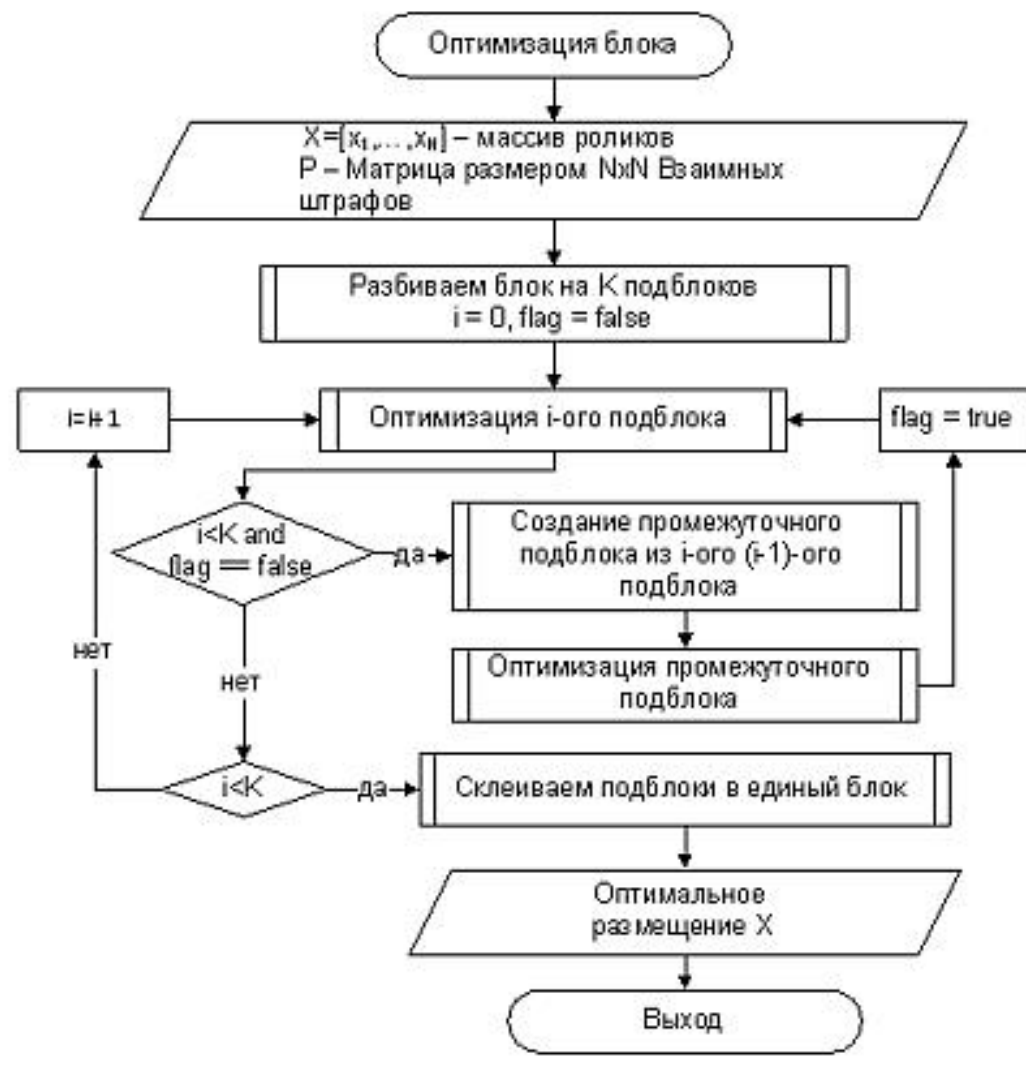

ис. 1.

ложность предложенного лгоритм в целом линейн и р вн $C * N / 30$, где - число опер ций, приходящихся н обр ботку одного подблок $\mathrm{p}$ змер не более 30 


\section{исленные исследов ния}

ценим численно точность и скорость р боты предложенного выше лгоритм . пределить точность р боты можно, зн я оптим льное значение

, которое можно получить лгоритмом полного перебор . виду большой ресурсоемкости т кого подход сдел ть это предст вляется возможным только для небольших $N$. езульт ты ср внительных исследов ний для $N \leq 10$ приведены в т блице $1 . \quad$ колонке « бъекты» в т блице 1 приведен кодировк н бор объектов, н котором проводился з мер точности р боты лгоритмов. пример, кодировк «1112223» обозн ч ет, что имеется объектов, которые поделены н 3 подгруппы: три один ковых объект «1», три один ковых объект «2» и объект «3». ждый объект конфликтует только с объект ми своей подгруппы с один ковым единичным штр фом. к к решение 3 д чи (2) н хождения минимум $W^{*}$ триви льно, в д нной 3 д че мы иск ли только минимум $W$ в 3 д че (1).

блии 1. н чения для р змещения 10 и менее объектов.

\begin{tabular}{|c|c|c|c|c|}
\hline & \multirow[t]{2}{*}{ бъекты } & \multirow{2}{*}{$\begin{array}{c}\text { лгоритм полного } \\
\text { перебор } . \text { Значение } \\
W_{\text {опт }}\end{array}$} & \multicolumn{2}{|c|}{$\begin{array}{c}\text { редложенный } \\
\text { лгоритм: }\end{array}$} \\
\hline & & & н чение & $W_{\text {алг }}$ \\
\hline 7 & 1112223 & 2.58 & 2.58 & \\
\hline 8 & 11122334 & 2.26 & 2.26 & \\
\hline 9 & 111122233 & 3.82 & 4.02 & \\
\hline 10 & 1111222334 & 3.35 & 3.52 & \\
\hline
\end{tabular}

ри больших зн чениях $N$ нет возможности н йти оптим льную р сст новку с помощью лгоритма полного перебора, но можно провести специ льные тесты с очевидным решением.

ест 1. озьмем множество из 100 объектов, которое состоит из двух подмножеств: $\left(x_{1}, x_{3}, x_{5} \ldots x_{99}\right),\left(x_{2}, x_{4}, x_{6} \ldots x_{100}\right)$, по 50 один ковых объектов в к ждом. іждый объект конфликтует только с объект ми своего подмножеств с один ковым единичным штр фом. удем иск ть минимум $W$ в 3 д че (1). птим льн я расстановка для д нного теста совп д ет порядком следов ния объектов $x_{1}, \ldots x_{100}$ или, другими слов ми, опгим льным р змещением будет являться чередование объектов из первого подмножеств

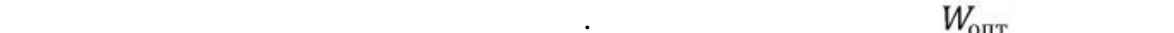
(1): и опт $\left(x_{1}, x_{2}, x_{3} \cdots x_{100}\right)={ }_{2} \cdot W_{\text {опт }}\left(x_{1}, x_{3}, x_{5} \cdots x_{99}\right)={ }_{2} \cdot$

$$
\left(\begin{array}{c}
\frac{1}{2}+\frac{1}{4}+\frac{1}{6}+\cdots+\frac{1}{98}+ \\
\frac{1}{2}+\cdots+\frac{1}{96}+ \\
+\cdots+ \\
+\frac{1}{2}
\end{array}\right)
$$

десь в первой строке запис н штр ф з конфликты объект $x_{1}$ и всех ост льных объектов первого подмножеств $\left(x_{3}, x_{5} \ldots x_{99}\right)$. о второй строке штр ф з конф.ликты $x_{3}$ и всех ост льных объектов первого подмножеств исключ я $x_{1}$, и т.д. осле преобразований формул (4), получим оптим льное зн чение функции штр เфа

$$
W_{\text {опт }}=2 \cdot \sum_{i=1}^{49} \frac{(50-i)}{2 \cdot i}=174.96
$$

рименяя к тому же входному множеству объектов предложенный лгоритм, получим $W_{\text {алг }}=175.86$.

ест 2. Возьмем н бор из 100 объектов $x_{1}, x_{2}, \ldots x_{100}$. Матрицу поп рных штр фов определим следующим обр зом:

$$
\begin{cases}p_{i j}=1, & \frac{|i-j|}{5} \in Z \\ p_{i j}=0, & \frac{|i-j|}{5} \notin Z\end{cases}
$$

Іругими словами, из первоначального множества $X$ конфликтуют между собой только элементы из 5-ти подмножеств: $\left(x_{1}, x_{6}, x_{11} \ldots x_{96}\right),\left(x_{2}, \ldots x_{97}\right)$, $\left(x_{3}, \ldots x_{98}\right),\left(x_{4}, \ldots x_{99}\right),\left(x_{5}, \ldots x_{100}\right)$. Оптим льная расстановка для данного теста совпад ет с порядком следования объектов $x_{1}, \ldots x_{100}$.

осчит ем оптим льный штр ф для д нного размещения. Ввиду того, что конфликтующие подмножеств один ковы с точки зрения штр ф, мы можем подсчит ть штр ф для одной группы, н пример $\left(x_{1}, \ldots x_{96}\right)$, и умножить его н 5. оспользов вшись определением (1), получаем: 
и опт $\left(x_{1}, x_{2}, \cdots \dot{x 100}\right)=5^{\cdot} \boldsymbol{n \text { опт } ( x _ { 1 } , x _ { 6 } \cdots x _ { 9 6 } ) = 5}$.

$$
\left(\begin{array}{c}
\frac{1}{5}+\frac{1}{10}+\frac{1}{15}+\cdots+\frac{1}{95}+ \\
\frac{1}{5}+\cdots+\frac{1}{90}+ \\
+\cdots+ \\
+\frac{1}{5}
\end{array}\right)
$$

десь в первой строке 3 пис н штраф за конфликты $x_{1}$ и всех ост льных объектов первого подмножеств $\left(x_{1}, x_{6}, x_{11} \ldots x_{96}\right)$. ] о второй строке штр ф за конфликты $x_{6}$ и всех ост льных объектов первого подмножеств , исключ я $x_{1}$, и т.д. осле преобр зований (4) получаем:

$$
W_{\text {опт }}=5 \cdot \sum_{i=1}^{19} \frac{(20-i)}{5 \cdot i}=51,95
$$

рименим к первон ч льному подмножеству предложенный лгоритм, получ ем $W_{\text {алг }}=52,76$.

ест 3. 'озьмем 34 объект, которые поделены н 2 неконфликтующие группы: 12 объектов первой группы конфликтуют между собой с штр фом $p=10$ и 22 объект 2-ой группы с штр фом $p=2$. птим льное $\mathrm{p}$ змещение в этом случ е будет иметь вид:

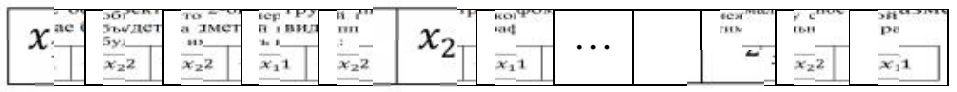

где $x_{1}$ - объект из 1-ой группы, $x_{2}$ - объект из 2-ой группы.

птим льное зн чение для т кого р змешения: $W_{\text {опт }}=186,12$.

редложенный лгоритм н ходит решение: $W_{\text {алг }}=188,0$.

ест 4. роведем тест н логичный тесту 2, только с 3-мя группами по 30

вз имно не конфликтующих объектов. Т.е. имеем объекты $x_{1}, x_{2}, \ldots x_{90}$. ункция поп рных штр фов:

$$
\begin{cases}p_{i j}=1, & \frac{|i-j|}{3} \in Z \\ p_{i j}=0, & \frac{|i-j|}{3} \notin Z\end{cases}
$$

птим льная расстановк для д нного тест совп д ет с порядком следов ния объектов $x_{1}, \ldots x_{90}$

$$
\boldsymbol{u}_{\text {опт }}=3 \cdot \boldsymbol{U}_{\text {опт }}\left(x_{1}, x_{4} \ldots x_{87}\right)=3 \cdot\left(\begin{array}{c}
\frac{1}{3}+\frac{1}{6}+\frac{1}{9}+\cdots+\frac{1}{87}+ \\
\frac{1}{3}+\cdots+\frac{1}{84}+ \\
+\cdots+ \\
+\frac{1}{3}
\end{array}\right)
$$

осле преобр зов ний получ ем:

$$
W_{\text {опт }}=3 \cdot \sum_{i=1}^{29} \frac{(30-i)}{3 \cdot i}=89,85
$$

рименим к первон ч льному подмножеству предложенный лгоритм, получ ем $W_{\text {алг }}=90,38$.

ри подготовке д нной ст тьи вторы пыт лись н йти худшие примеры, в

\begin{tabular}{|c|c|c|c|c|c|}
\hline $\begin{array}{l}\text { № } \\
\text { пример }\end{array}$ & $W_{\text {опт }}$ & $\longrightarrow$ & $\begin{array}{l}-\quad- \\
\frac{W_{\mathrm{a}} \frac{\mathrm{Ir}}{}-W_{\mathrm{or}}}{W_{\mathrm{agr}}} * 100\end{array}$ & 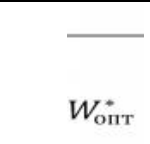 & 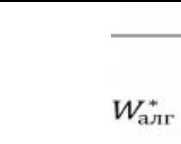 \\
\hline 1 & 174.96 & 175.86 & 0,51 & 0,0 & 3,0 \\
\hline 2 & 51,95 & 52,76 & 1,54 & 0,0 & 0,0 \\
\hline 3 & 186,12 & 188,0 & 1,00 & 18,0 & 18,00 \\
\hline 4 & 89,85 & 90,38 & 0,59 & 0,0 & 0,0 \\
\hline
\end{tabular}
которых зн чение ЦФ предложенного лгоритм зн чительно отлич лось бы от оптим льного значения , но это ок з лось не т к просто. езульт ты численных исследований решения оптимиз ционной $з$ д чи (1) для $W$ и з д чи (2) для $\quad W^{*}$ приведены в т блице

бл. 2. водн ят блиц результ тов численных исследов ний.

корость р боты предл г емого лгоритм в секунд х для больших $N$ приведен н гр фике, изобр женномн рис. 2 . 


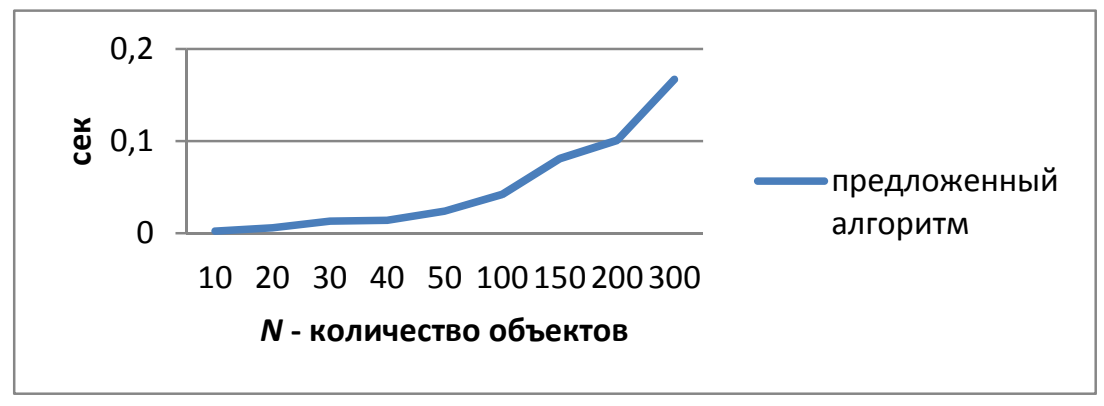

ис. 1. висимость времени р боты предложенного лгоритм от количесте объектов

ложность предложенного лгоритм упорядочения всех конфликтующих объектов в целом линейн и р вн $O(N)$.

\section{ключение}

ст тье построены лгоритмы решения 3 д ч оптим льного упорядочения конфликтуюших объектов. ссмотрены 3 д чи двух типов: когд штр фуется только непосредственное соседство объектов (з д ч TSP), и обобщенный случ й, когд штр ф действует к к н соседние, т к и н отд ленные друг от друг конфликтующие объекты, и убыв ет в 3 висимости от числ p сположенных между ними объектов. ок 3 н связь 3 д чи оптим льного упорядочения конфликтующих объектов с 3 д чей коммивояжер . ссмотрен 3 д ч TSP c p зреженной м трицей штр фов. ля н иболее p спростр ненных случ ев т кой 3 д чи TSP, когд м триц штр фов имеет вид ленточной или блочно-ди гон льной, построены точные быстрые лгоритмы, достиг ющие миним льного нулевого зн чения целевой функции. ок 3 но необходимое и дост точное условие, при котором достиг ется нулевой минимум в т ких 3 д ч х TSP. редложенные лгоритмы эффективны для $\mathrm{p}$ зреженных м триц. $\mathrm{x}$ т кже можно успешно применять в к честве приближенных лгоритмов к м триц м близким к ленточным, блочно-ди гон льным и р зреженным. ля общего случ я в ст тье предложен эвристический лгоритм, который пок з л высокое быстродействие при незн чительных потерях в к честве решения. ля з д чи TSP (2) он т кже p бот ет эффективно. д лось добиться хорошей его м сшт бируемости до произвольного р змер при сохр нении линейной сложности, что позволило н м использов ть предложенные лгоритмы н пр ктике н больших объем х д нных, н пример, для з д ч р змещения рекл мных 3 к зов в сетях . х можно применять т кже для решения з д ч коммивояжер и близких з д ч обход гр фов, в том числе в социологии, при н хождении оптим льных путей в соци льных сетях.

\section{писок литер туры}

[1]. узюрин . ., омин . . ффективные лгоритмы и сложность вычислений: чебное пособие. $-\quad . \quad$, 2007. - 312 c.

[2]. http://en.wikipedia.org/wiki/Travelling_salesman_problem

[3]. Peter Komjach: http://mathoverflow.net/questions/30270/maximum-number-ofmutually-equidistant-points-in-an-n-dimensional-euclidean-spac, geometry - Maximum number of mutually equidistant points in an n-dimensional Euclidean space is $(n+1)$ Proof - MathOverflow, answered Jul 2, 2010.

[4]. жордж ., ю ж. исленное решение больших р зреженных систем ур внений: ер. с нгл. - $\therefore$ ир, 1984. -333 с.

[5]. исс нецки . ехнология р зреженных м триц: ер. с нгл. - .: ир, 1988. $410 \mathrm{c}$.

[6]. ормен ., ейзерсон ., ивест ., т йн . лгоритмы: построение и н лиз: од ред. р сиков . . - 2-е изд. -.$\therefore$ ильямс, 2005. - 1296 с. 


\title{
Optimal Ordering of Conflicting Objects and the Traveling Salesman Problem
}

\author{
Alexey Voevodin, Semen Kosyachenko \\ Silver-AVV@yandex.ru,spiero@yandex.ru \\ Business Center «Video International», Moscow, Russia
}

\begin{abstract}
The paper presents the setting of the problem of optimal ordering of conflicting objects. This problem appears in sociology, in advertising on TV and other media networks. Point is that positions of some conflicting commercials were as far apart as possible if commercials belong to the same class of goods or the same advertiser or brand. Solution algorithms are described for this and related problems such as the Travelling Salesman Problem (TSP). The tasks are considered of two types: when penalized only a juxtaposition of objects according to the TSP, and the generalized case when the penalty acts on the neighboring and on the distant to each other conflicting objects. The penalty depends on the number of objects that lie between them. The TSP with sparse matrix is also considered. For sparse practice cases of the TSP with the band or block diagonal matrix necessary and sufficient conditions are proved to objective function attained its zero minimum and the algorithms guaranteeing exact solution for the TSP are constructed. The proposed algorithms are effective also for sparse matrices of general type. These algorithms can also be successfully used as approximate algorithms for matrices close to the band or block diagonal. For the general case in the paper we propose a heuristic algorithm with high performance and good accuracy of solution. We got good scalability to any size while maintaining linear complexity, which allowed us to use these proposed algorithms in practice for large amounts of data, such as advertising in media networks. The practical results of analytical and numerical investigations of algorithm complexity and solution accuracy are presented.
\end{abstract}

Keywords: optimal placement; Travelling Salesman Problem; TSP; NP-hard problems; band matrix; sparse matrix; greedy algorithm; penalty function; conflicts, media network; advertising.

\section{References}

[1]. Kuzyurin N.N., Fomin S. . Effektivnye algoritmy i slozhnost' vychislenij [Efficient algorithms and computational complexity]. Uchebnoe posobie [Tutorial]. Moscow, MIPT' Publ., 2007. 312 c.

[2]. http://en.wikipedia.org/wiki/Travelling_salesman_problem

[3]. Peter Komjach: http://mathoverflow.net/questions/30270/maximum-number-of-

mutually-equidistant-points-in-an-n-dimensional-euclidean-spac, geometry - Maximum number of mutually equidistant points in an $n$-dimensional Euclidean space is $(n+1)$ Proof - MathOverflow, answered Jul 2, 2010.

[4]. Alan George, Joseph W. H. Liu. Computer Solution of Large Sparse Positive Definite Systems. Prentice-Hall, Inc., Englewood Cliffs, N.J. 1981 - 324 pages.

[5]. Sergio Pissanetzky. Sparse Matrix Technology. Academic Press, 1984 - 321 pages.

[6]. Cormen, Thomas H.; Leiserson, Charles E., Rivest, Ronald L., Stein, Clifford. Introduction to Algorithms (3rd ed.). MIT Press and McGraw-Hill. 2009 [1990]. 